\title{
Acoustic metasurface by layered concentric structures
}

\author{
Shanjun Liang, ${ }^{1,2}$ Tuo Liu $\odot,{ }^{2,}{ }^{*}$ He Gao, ${ }^{2}$ Zhongming Gu, ${ }^{2}$ Shuowei An, ${ }^{2}$ and Jie Zhu $\odot^{2,3, *}$ \\ ${ }^{1}$ Division of Science, Engineering and Health Studies, College of Professional and Continuing Education, The Hong Kong Polytechnic \\ University, Hung Hom, Kowloon, Hong Kong SAR, China \\ ${ }^{2}$ Department of Mechanical Engineering, The Hong Kong Polytechnic University, Hung Hom, Kowloon, Hong Kong SAR, China \\ ${ }^{3}$ The Hong Kong Polytechnic University Shenzhen Research Institute, Shenzhen 518057, China
}

(Received 9 July 2020; revised 2 September 2020; accepted 14 October 2020; published 11 December 2020)

\begin{abstract}
Metasurface-based acoustic wave-front manipulation with broad bandwidth and low transmission loss shows great significance in high-intensity applications such as ultrasonic therapy, acoustic tweezers, and haptics. By taking advantage of the helical-structured metamaterials and their concentrically layered arrangement, we present a systematic strategy to construct two-dimensional transmissive acoustic metasurfaces that possess matched impedance to the background medium and simple governing parameters. As a proof of concept, a concentrically layered circular metalens supporting conversion from spherical wave to plane wave is designed and experimentally demonstrated. It is capable of operating in more than one octave band with high transmission. This work could inspire more intriguing and flexible designs in three-dimensional wave control, which may enhance the practicality of acoustic metasurfaces.
\end{abstract}

DOI: 10.1103/PhysRevResearch.2.043362

\section{INTRODUCTION}

Realizing acoustic wave control with artificial materials is one of the central topics in material science. In the past decades, acoustic metamaterials [1,2], assemblies of subwavelength meta-atoms that collectively behave like continuous materials with unconventional properties, bring new thoughts to both material design and wave physics. Particularly, an emerging and promising direction is their two-dimensional (2D) counterparts known as metasurfaces [3], which have shown great potential due to not only the extraordinary functionalities but also the subwavelength thickness. Unlike the refractive lenses constructed using gradient-index phononic crystals $[4,5]$ or metamaterials [6-8], metasurfaces are diffractive devices capable of modulating the wave front via the generalized Snell's law [9] and phase engineering and have enabled many applications.

Two major types of building blocks for acoustic metasurfaces have been developed and investigated in recent years, including Helmholtz resonator arrays [10-13] and spacecoiling structures [14-19]. They can generate phase delay in a $2 \pi$ range either for transmitted or reflected waves, allowing a wealth of functionalities based on wave-front modulation. Among them, the resonance-based structures such as Helmholtz resonator arrays, generally suffers from limited range of working frequency due to their drastic change of phase shift. Metasurfaces composed of this type

\footnotetext{
*Corresponding authors: tuoliu@ @olyu.edu.hk; jiezhu@polyu.edu.hk

Published by the American Physical Society under the terms of the Creative Commons Attribution 4.0 International license. Further distribution of this work must maintain attribution to the author(s) and the published article's title, journal citation, and DOI.
}

of building blocks, therefore, show excellent performance of wave-front modulation only at specific operating frequencies [11]. The other type, the coiling-up space structures elongate the acoustic wave's traveling path inside the structure to provide phase delay. An optimization of coiling-up space is the helical-structured metamaterials [20-22] that possess higher space-utilization rate and dispersion-free properties. This makes them an ideal candidate for broadband use. Moreover, by introducing gradient impedance matching layers [23-29], periodic valleys in transmission spectra can be "pulled up", leading to a higher energy efficiency in a broad bandwidth.

The unit cells mentioned above usually have a rectangular shape. When it comes to 3D wave control, especially for centrally symmetric cases, the large number of unit cells is proportional to the area of metasurfaces [30]. This increases the workload of design and fabrication. An efficient way to simplify the process is the utilization of circular unit cells, as has been demonstrated by the acoustic Fresnel lens [31] and some concentric metasurfaces [32-35]. These devices make good use of symmetry in cylindrical coordinate system yet are still restricted to narrow operating bandwidth.

In this work, we introduce helicoids with gradient impedance to the cylindrical layered unit cells to obtain a broadband metasurface with high energy transmission efficiency and easy-controlled effective parameters. Taking advantages of the helical structures, the effective index and impedance can be analytically determined from the geometry of the unit cells. Based on this, a broadband wave-front converter is designed and experimentally demonstrated in more than one octave band.

\section{THEORY AND DESIGN}

Composed by concentric ring-shaped unit cells, a circular metalens is presented in this work to demonstrate the design 


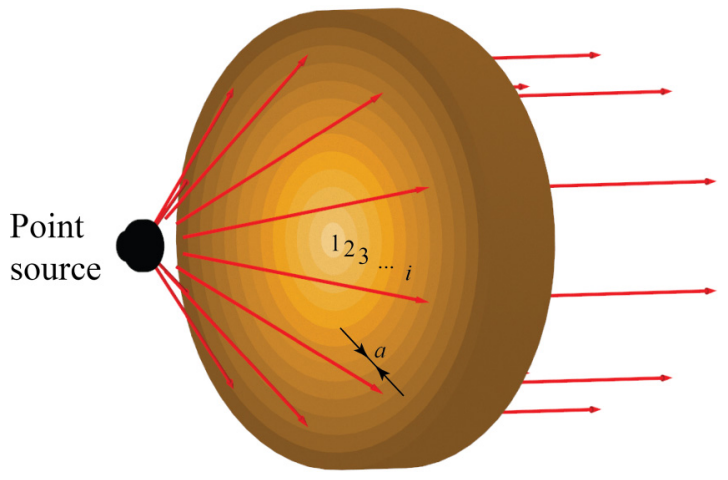

FIG. 1. Cylindrical layered metasurface. The metasurface is constructed by a series of concentric circular layers with a uniform thickness $a$ and different effective indices.

and mechanism of the circumferentially symmetric metasurface. As depicted in Fig. 1, incidence along the $z$ axis is considered. In the radial direction, there are a series of circular layers with uniform thickness $a$. The effective refractive index varies only in the radial direction and can be determined separately in each layer. Such a metalens can be designed layer by layer and used for symmetric wave control in 3D space. The layered unit cell occupies the space between two cylindrical shells. When putting a polar array of bending blades between two neighboring cylindrical shells, the obtained tubelike traveling path is longer than the height of the unit cell, $h$, leading to an increased effective refractive index for waves traveling along the $z$ direction [see Fig. 2(a)]. As reported in previous research $[20,26]$, helical-structured bending blades have very low dispersion and high space utilization rate, which is an ideal building block for our acoustic metalens. The point $(x, y, z)$ on the $n$th blade can be described by the expression

$$
\begin{aligned}
x & =\rho \cos \left[\frac{l}{r} \frac{z}{h}+(n-1) \phi\right], y=\rho \sin \left[\frac{l}{r} \frac{z}{h}+(n-1) \phi\right], \\
r_{i-1} & <\rho<r_{i}, 0<z<h,
\end{aligned}
$$

where the origin is located at the bottom center shown in Fig. 2(b), $\rho$ and $z$ are independent variables along the radius and central axis of the unit cell, respectively, $r_{i-1}$ and $r_{i}$ are the inner and outer radius of a unit cell with $r=\left(r_{i-1}+r_{i}\right) / 2, i$ denotes the $i$ th unit cell from the center (see Fig. 1), $h$ is the height of the unit cell shown in Fig. 2(a), and $\phi=2 \pi / N$ is the rotating angle interval in a polar array of $N$ blade copies. Here $l$ represents the projected length of the unwrapped blue line along the $x$ axis.

The acoustic behavior of those tortuous tubes in each layer can be described by a 2D model as shown in Fig. 2(b). The expression of an individual leading thread (a helix) can be obtained by setting $\rho=r$ in Eq. (1). Unwrapping two adjacent leading threads to a plane surface, one can obtain two parallel straight lines. Comparing the plane regions I and II in Fig. 2(b), the unwrapped threads form a narrow path that is longer than $h$. Hence, the effective refractive index is written as

$$
N_{\text {eff }}=1 / \cos \theta,
$$

where $\theta$ is the completion of the helix angle. When $\theta$ approaches zero, the helical blades become parallel to the $z$ axis, and the effective index is close to that of the background medium. According to the theory of wave propagation in the tube with varying cross section, the ratio of impedance between the effective medium and background medium takes the (a)
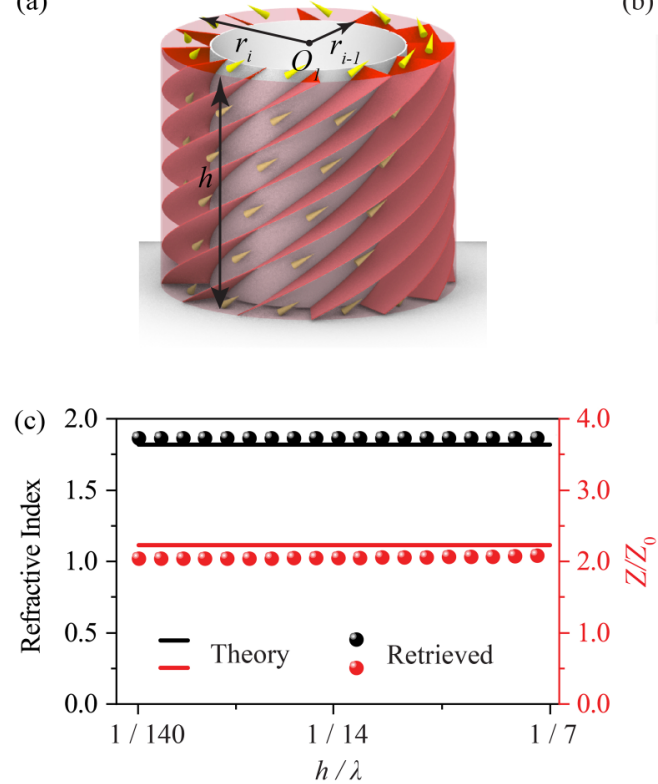

(b)
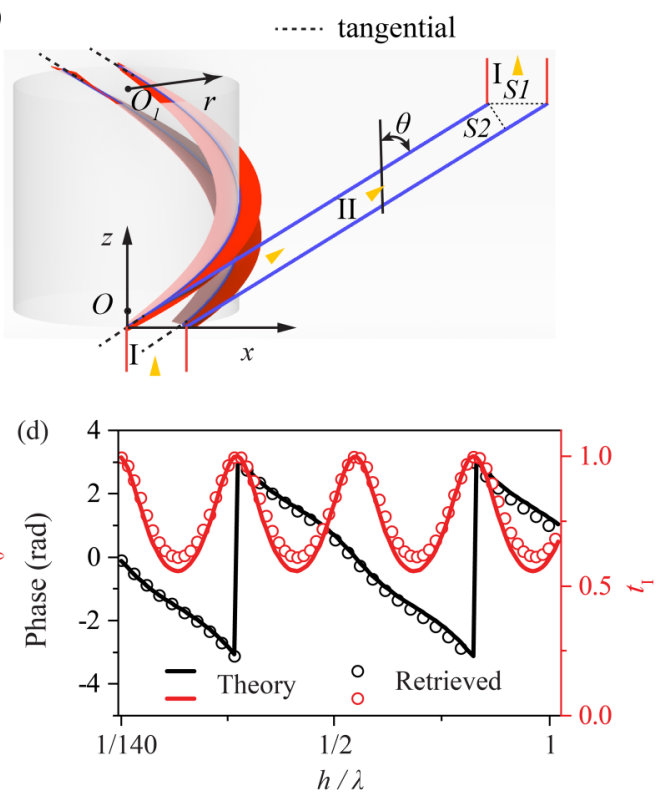

FIG. 2. Unit cell with uniform helix angle. (a) Construction of a cylindrical layered unit cell. The yellow arrow indicates the traveling path of sound wave. The height $h$ is $50 \mathrm{~mm}$. The thickness of the blade is $0.8 \mathrm{~mm}$. The inner and outer radius $r_{i-1}$ and $r_{i}$, are 20 and $30 \mathrm{~mm}$, respectively. (b) Schematic illustration of the formation of the blades by winding straight lines around a cylinder. $\theta$ is the complement angle of the helix. (c) Retrieved effective parameters and (d) transmission properties of a typical unit cell. The scatters and lines are simulation and theoretical results, respectively. The unit cell's governing parameter $l=83 \mathrm{~mm}$. 
(a)

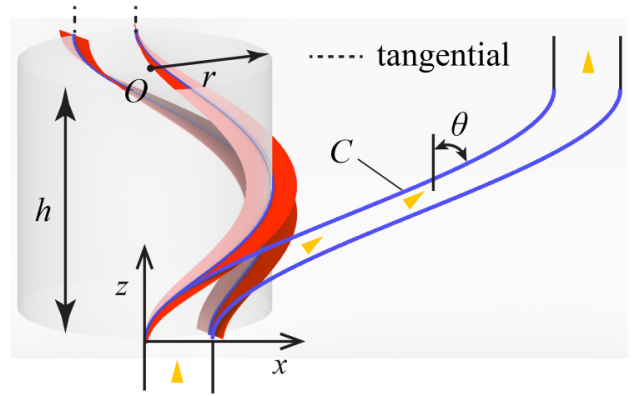

(b)

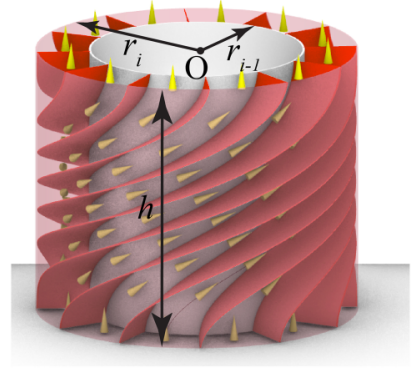

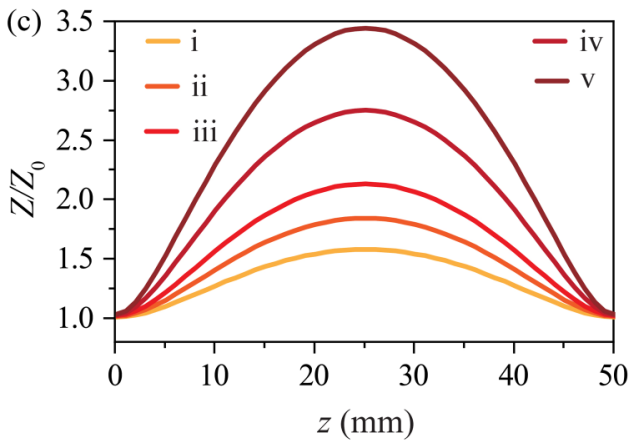

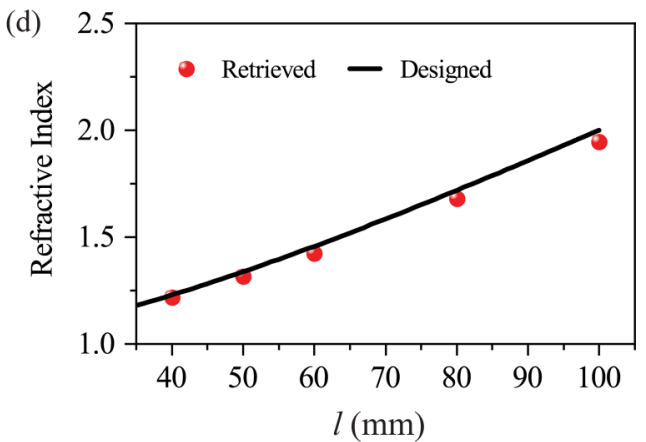

FIG. 3. Impedance-matched unit cell. (a) Schematic illustration of the formation of the blades by winding cubic Hermite splines around a cylinder. (b) Schematic illustration of wave propagation in the unit cell. $h, r_{i-1}$, and $r_{i}$ are same as that of the unit cell in Fig. 2. (c) Distribution of the effective impedance ratio along the $z$ axis. From . i to v, relating parameters $l$ of the unit cell are 40, 50, 60, 80, and 100 mm, respectively. (d) Refractive index as a function a $l$. The solid line is obtained from Eq. (8). The scatters denote the retrieved effective index of the unit cells in (c).

form $Z / Z_{0}=s_{1} / s_{2}=1 / \cos \theta$, where $s_{1}$ and $s_{2}$ are the widths of the $2 \mathrm{D}$ tube shown in Fig. 2(b). Meanwhile, the thickness $t$ of the helical blades needs to be considered to modify $Z / Z_{0}$, yielding

$$
\frac{Z}{Z_{0}}=\frac{1}{\cos \theta-\frac{N t}{2 \pi r}} .
$$

To make sure the effective parameters are valid, the wavelength is set to be more than seven times of $h$. The theoretical results obtained from the geometric model and the retrieved parameters [36,37] show good agreement in Fig. 2(c), indicating a dispersion-free property in the low frequency regime. Furthermore, the transmission properties of the structure, i.e., the phase and energy transmission coefficient, can be determined by the theoretical effective parameters even though the wavelength is comparable to the height of the unit cell. Therefore, the helical structures in the cylindrical layers exhibit dispersion-free property in a wide frequency range. Note that the effective model makes sense when the frequency of incidence is lower than the cutting-off frequency of the helical tube. In detail, substituting the longer edge length of the square tube $a=0.01 \mathrm{~m}$ and sound velocity $c_{0}=343.2 \mathrm{~m} / \mathrm{s}$ into $f_{c}=c_{0} / 2 \sqrt{a^{-2}}$, the cutting-off frequency $f_{c}$ is $17160 \mathrm{~Hz}$, much higher than the designed working frequency. It is also worth noting that the shape of the blade is governed only by the helix angle $\theta$, which means the property of the unit cell is determined by the leading threads. This brings convenience to the control of effective impedance.

In view of the fact that a uniform helix angle leads to the impedance mismatching between the unit cell and the back- ground medium at both ends, one needs to optimize the shape of the leading threads for a gradually distributed impedance along the $z$ axis. A perfectly matched impedance requires two conditions: (1). $\theta=0$ when $z=0$ and $z=h$; (2). The blades' surfaces always have a smooth and gradual shape. An inner blade meeting the conditions is designed to be

$$
\begin{aligned}
x & =\rho \cos \left(\frac{l}{r}\left(\frac{z}{h}\right)^{2}\left(3-2 \frac{z}{h}\right)\right), \\
y & =\rho \sin \left(\frac{l}{r}\left(\frac{z}{h}\right)^{2}\left(3-2 \frac{z}{h}\right)\right), \\
r_{i-1} & <\rho<r_{i}, 0<z<h,
\end{aligned}
$$

where $l$ is the only parameter used to control the shape of a blade. Similar to the unit cell with uniform helix, $l$ represents the projected length of the Hermite spline along the $x$ axis. The larger it is, the more tortuous structure can be obtained, which means a lower equivalent sound speed in the $z$ direction and a larger effective index. To show the variation of the effective impedance along the $z$ axis, we need to find the angle $\theta$ with respect to $z$. To begin with,

$$
\tan \theta=d x / d z
$$

The curve $C$ in Fig. 3(a) can be described by a parametric equation in the $x-z$ plane of a local coordinate system

$$
x=l\left(\frac{z}{h}\right)^{2}\left(3-2 \frac{z}{h}\right) .
$$

Here Eq. (6) is one type of the cubic Hermite spline [38]. Combining Eqs. (3), (5), and (6), the resultant impedance 
(a)
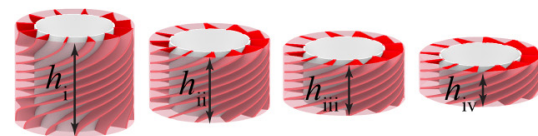

(b)

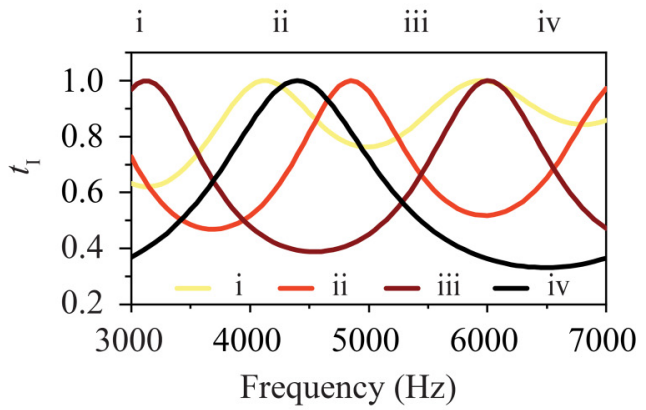

(c)

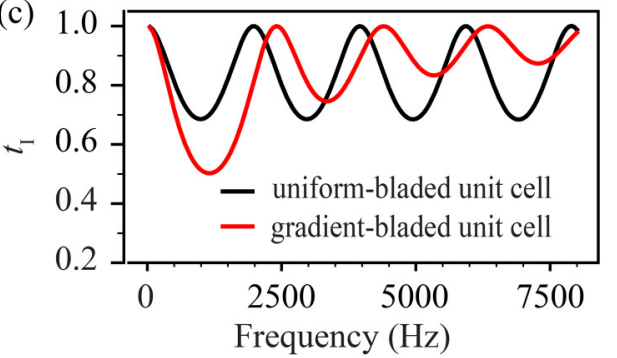

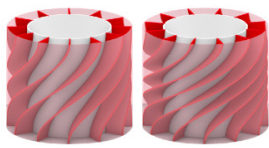
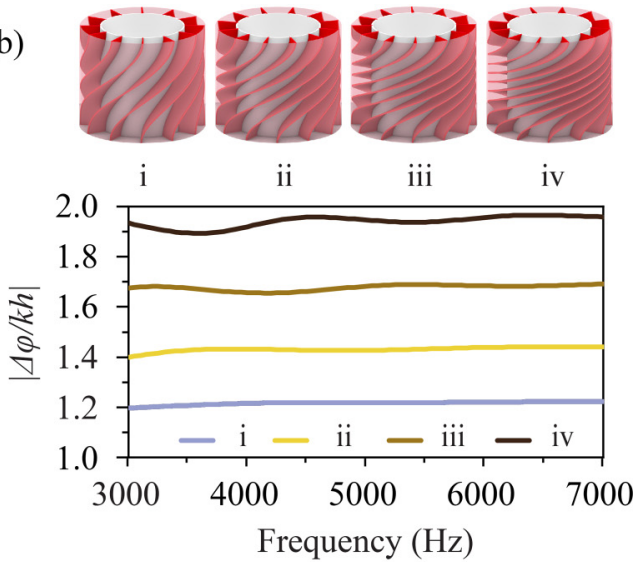

(d)

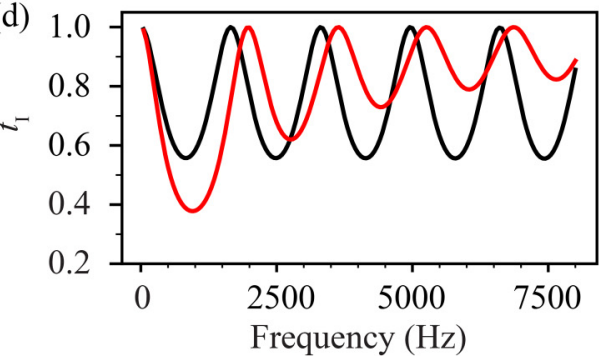

FIG. 4. Broadband performance of the gradient unit cells. (a) Transmission coefficients of the truncated unit cells. The values of $h_{i}$ to $h_{i v}$ are 50,34, 26, and $18 \mathrm{~mm}$, respectively. Structures ii to iv are obtained by cutting down the same length at both ends of the original unit cell. (b) Broadband phase delays for different unit cells. From i to iv, governing parameters $l$ are 40, 60, 80, and 100 mm, respectively. (c),(d) Comparison of the overall transmission coefficients of the uniform and gradient unit cells. The governing parameter $l=80 \mathrm{~mm}$ in (c) and $l=100 \mathrm{~mm}$ in (d), respectively. (c) and (d) share the same legend.

varies as a function of $z$ and is given by

$$
\frac{Z(z)}{Z_{0}}=\frac{1}{\left[\left(\frac{6 l z}{h^{2}}\left(1-\frac{z}{h}\right)\right)^{2}+1\right]^{-\frac{1}{2}}-\frac{N t}{2 \pi r}} .
$$

The tangential of the gradient helical surface is parallel to the $z$ axis when $z=0$ and $z=h$. As can be seen in Fig. 3(c), this leads to a nearly perfect impedance matching between the unit cell and the background medium on the entrance and exit surface. The maximum of impedance appears in the middle of the unit cell along the $z$ axis. Similar to the uniform helix model, the elongation of wave traveling path determines the effective index, which can be expressed as

$$
N_{\text {eff }}=\frac{1}{h} \int_{0}^{h} \sqrt{\left[\frac{6 l t}{h^{2}}\left(1-\frac{t}{h}\right)\right]^{2}+1} d t .
$$

Figure 3(d) illustrates that, based on the geometric relationship of Eq. (8), the effective index is governed only by $l$. The retrieved indices of the unit cells at a low frequency $(2000 \mathrm{~Hz})$ are all on the curve of Fig. 3(d), indicating the validity of adjusting the effective index by changing $l$.

The broadband performance of a unit cell of the designed properties is further investigated. First, the impedance
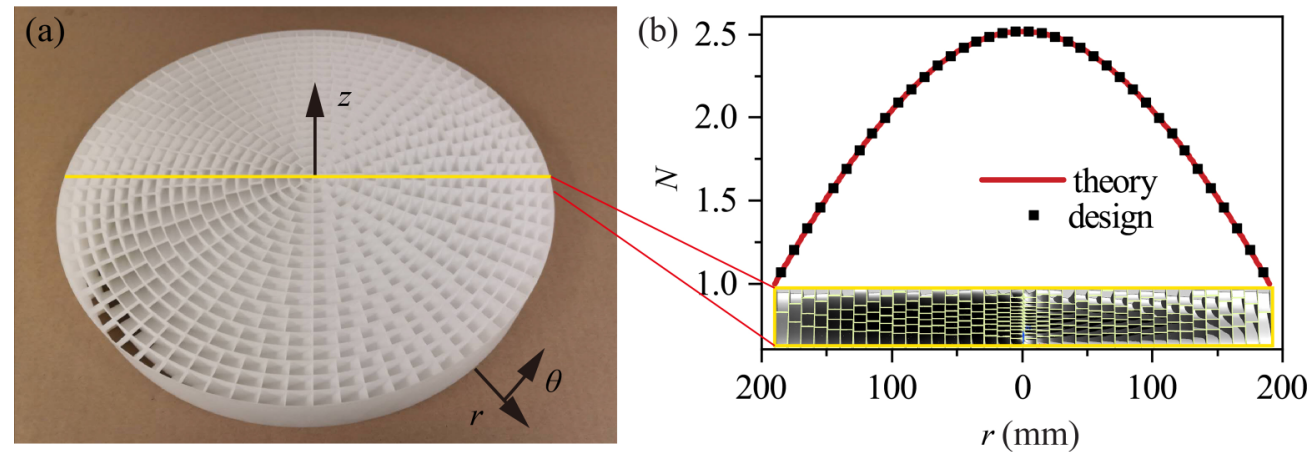

FIG. 5. Fabricated broadband wave-front converter sample. (a) Photograph of the sample. It has 19 layers, and the thickness of each layer is $10 \mathrm{~mm}$. (b) Designed index profile for the spherical to plane wave transformation. The yellow box shows the cross-sectional surface of the sample along its diameter. The red line with legend theory represents the required distribution of index obtained from Eq. (13). The black dots represent the discrete indices for each layer. 


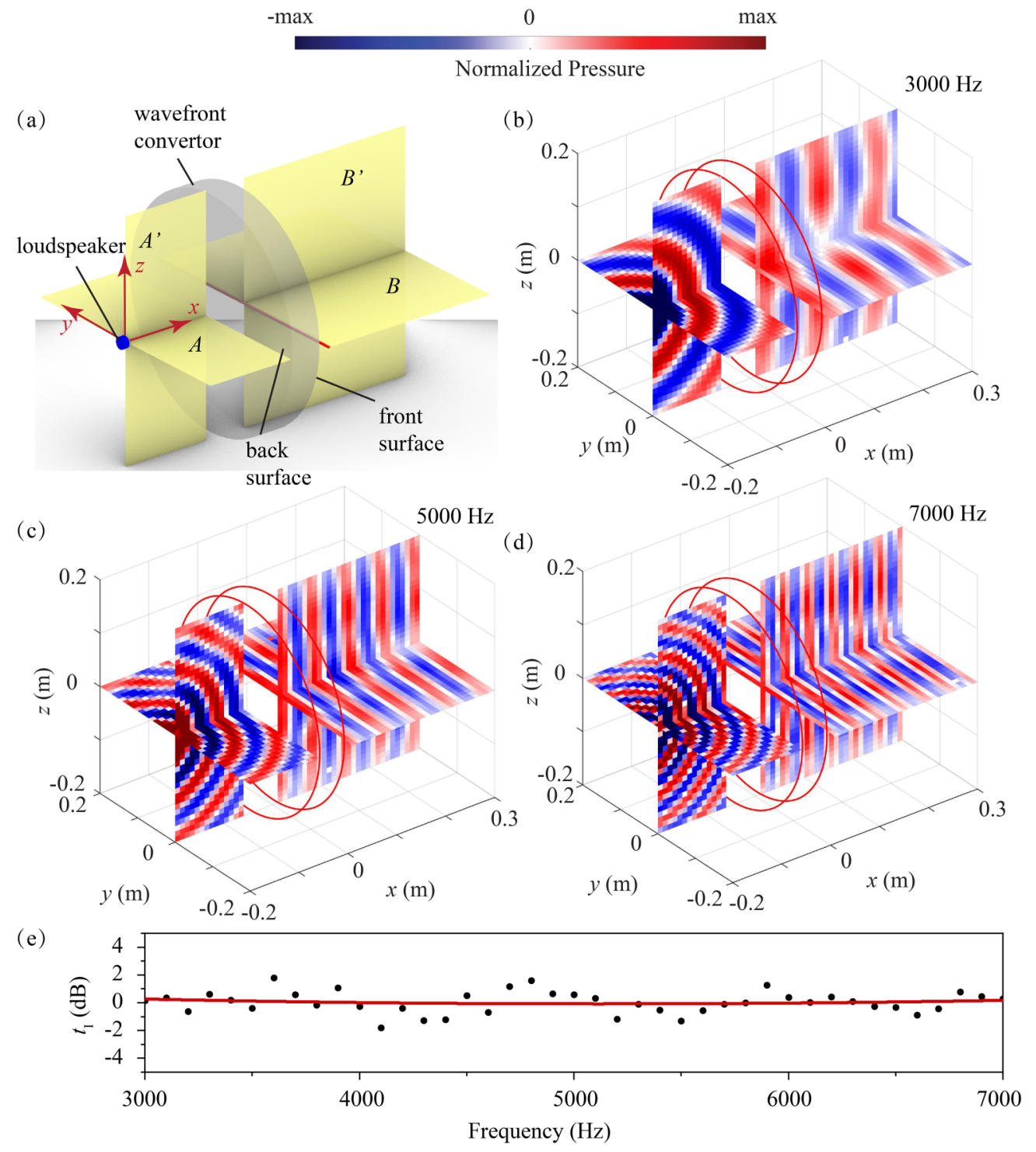

FIG. 6. Measurement of the lens. (a) illustrates the experiment setup and measured region. The metasurface-based wave-front convertor is a plate with a diameter of $380 \mathrm{~mm}$ and thickness of $50 \mathrm{~mm}$. The loudspeaker is located at the center of the lens $200 \mathrm{~mm}$ to its back surface along the $x$ axis. The tested region (yellow) are $140 \times 400 \mathrm{~mm}^{2}$ and $290 \times 400 \mathrm{~mm}^{2}$. The nearest distance between the microphone and the surface of the lens is $10 \mathrm{~mm}$. The data of surface $A^{\prime}$ is obtained in the absence of the metasurface. (b)-(d) illustrate the normalized pressure field at three frequencies, respectively. All three subfigures share the same normalization range. (e) Energy transmission coefficient of the lens.

matching near both ends can be verified by comparing the energy transmission spectra of the modified unit cells. When cutting down the region for impedance matching on both ends, the valley of the transmission coefficient becomes lower and lower, which is shown in Fig. 4(a). Comparing $i$ and iv, one may easily find that the impedance matching region enhances the energy transmission efficiency of the structure over spectrum. Second, the frequency dependency of the dynamic index characterizes the structures' dispersion behavior. The nearly flat curves in Fig. 4(b) thus confirms the dispersion-free property of the unit cells. Meanwhile, the slight fluctuation related to large $l$ values tells the trade-off between the index and the dispersion-free characteristic. Intuitively, the fluctuation of dynamic indices results from the limited impedance-matching length in comparison with the wavelength. Furthermore, the impedance matching property can be investigated by comparing the transmission spectra of the uniform and gradient unit cells. Figures 4(c) and 4(d) show that, for the uniform unit cells, as the frequency goes higher, the minimum values of the transmission remains constant, whereas their gradient counterparts have larger minimum values at higher frequencies despite a deeper first valley than that of the uniform unit cells. In fact, in the low frequency regime, the wavelength is too long to "recognize" the gradient impedance-matching layers. As the effective impedance in the middle of the gradient unit cell is larger than that of the uniform counterpart, the transmission coefficient has lower values at the first valley. In the high frequency regime, the shorter the wavelength is, the better transmission efficiency can be achieved by impedance matching. 


\section{A BROADBAND WAVE-FRONT CONVERTER}

A spherical wave front can be naturally generated by a point source, which is extensively used in practical scenarios. In the following, a spherical-to-plane wave converter is realized to demonstrate the design strategy and the performance of the planar lens. Considering spherical waves emanating from a point source to the incident plane of the lens, the incident phase along the $r$ direction is

$$
\varphi_{i}(r)=\varphi_{s}-k \sqrt{r^{2}+d^{2}},
$$

where $\varphi_{s}$ is the initial phase of the source, $k$ is the wave number, $d$ is the distance between the source and the incident plane of the lens. For the plane wave on the exit plane, the phase distribution $\varphi_{o}(r)$ is subjected to

$$
\varphi_{o}\left(r_{1}\right)-\varphi_{o}\left(r_{2}\right)=0,
$$

where $r_{1}$ and $r_{2}$ are two arbitrary positions along the $r$ axis.

For an impedance-matched planar lens free of local resonance, the phase delay is determined by its thickness $h$ and effective index $n(r)$, i.e.,

$$
\varphi_{o}(r)-\varphi_{i}(r)=-k h n(r) .
$$

Substituting Eq. (11) back into Eq. (10), then combining Eq. (9), the required refractive index can be obtained:

$$
n\left(r_{2}\right)=n\left(r_{1}\right)-\frac{\sqrt{r_{2}^{2}+d^{2}}-\sqrt{r_{1}^{2}+d^{2}}}{h} .
$$

In Eq. (12), let $r_{1}=0$ and $r_{2}=r$, accordingly,

$$
n(r)=n(0)-\frac{\sqrt{r^{2}+d^{2}}-d}{h} .
$$

With a given index $n(0)$ at the center of the lens, the index distribution can be uniquely determined. Figure 5(b) shows a possible case by setting $d=200 \mathrm{~mm}$ and $n(0)=2.516$. The discretization interval in Fig. 5(b) is the width of the layer, which is $10 \mathrm{~mm}$. Thus, for each layer, once finding the $l$ based on the required index, one can determine the shape of the blades and the structure of the layer as well.

With the geometrical model of the lens, a flat lens was manufactured using 3D printing technique. We conducted experiments to test its performance in the frequency range between 3000 and $7000 \mathrm{~Hz}$. The source is a loudspeaker with a radius of $14 \mathrm{~mm}$. The distance between the source and the lens is set to be $200 \mathrm{~mm}$. A scanning measurement was performed in a rectangular region on the output side of the lens. Figure 6(a) gives the experimental setup. The pressure patterns in Figs. 6(b)-6(d) display the formation of 3D plane waves in a broad bandwidth. The spherical wave front from a point source becomes plane wave front after passing through the lens. The pressure fields are normalized simultaneously, demonstrating the uniform performance of the device. To evaluate the energy transmission efficiency, we compare the transmitted sound fields with and without the lens. The contrast ratio between these two cases is defined as $t_{\text {lens }}=20 \log _{10}\left(I_{2} / I_{1}\right)$, in which $I_{1,2}$ is the integration of sound intensity along the diameter line in the presence (absence) of the lens. In Fig. 6(e) the energy transmission ratio is much larger than $-3 \mathrm{~dB}$ over the spectrum. Moreover, at some
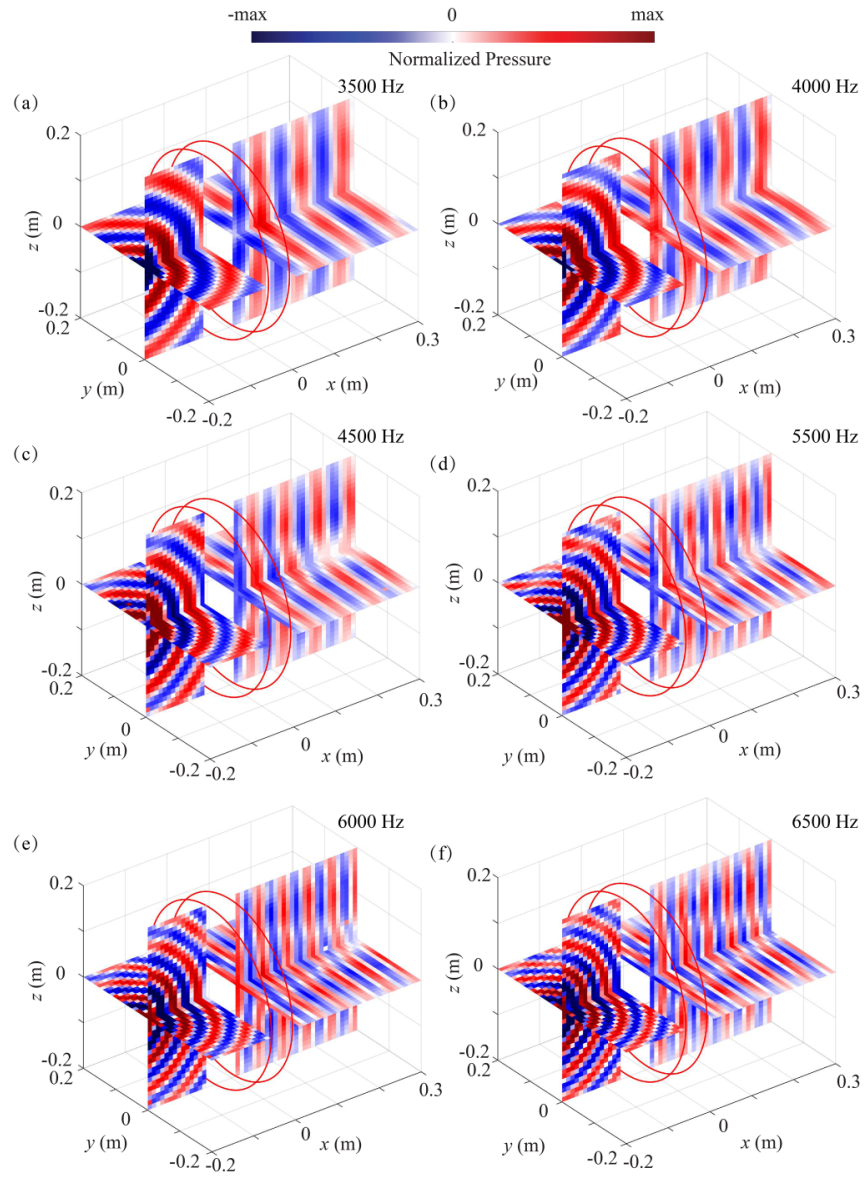

FIG. 7. Measured sound pressure fields of the wave-front converter at other frequencies. (a)-(f) Experimental results at some representative frequencies from 3500 to $6500 \mathrm{~Hz}$. The normalization of the data is based on same scale.

frequencies the transmission coefficient is over $0 \mathrm{~dB}$. The reason is that the lens limits the expansion of the spherical wave front.

\section{CONCLUSION}

In this work, a design strategy of concentrically layered lens has been proposed. The building blocks are generalized helical-structured metamaterials with gradient geometry along the perpendicular direction, possessing matched impedance with the background medium at both ends, nearly dispersion-free properties, and high transmission efficiency for more than one octave band. We have shown that the structures can be analytically described in a simple way. With effective properties being governed by only one geometrical parameter, it significantly reduces the difficulty of inverse design. A planar lens capable of transforming spherical wave to plane wave in a broadband has been designed and experimentally demonstrated following the strategy. Such a layer-by-layer strategy is helpful for the construction of complex index distribution, applicable to other flexible and broadband acoustic devices to enhance the competitiveness of metamaterial over conventional alternatives. In particular, the procedure could be employed to design efficient 

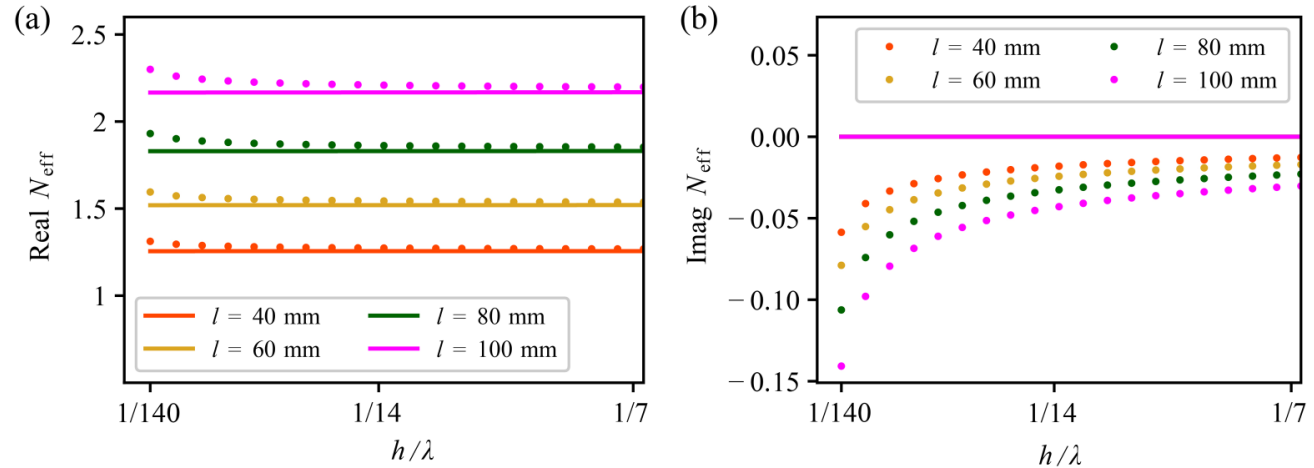

FIG. 8. Retrieved effective refractive index of the uniform spiraling units. (a) The real part of the effective indices. The solid lines represent the lossless cases, and the dotted markers denote the results with the inherent thermal and viscous losses being taken into account. (b) The imaginary part of the effective indices. The magenta solid line indicates the superposition of four colored lines corresponding to the lossless cases.

wave-front modulator in the field of acoustic imaging, soundmatter interaction, and acoustic haptics.

\section{ACKNOWLEDGMENTS}

This work was supported by National Natural Science Foundation of China (Grant No.11774297) and Research Grant Council of Hong Kong (Grant No. 152119/18E). T. L. acknowledges support from Internal Research Fund of The Hong Kong Polytechnic University (Grant No. ZZLC).

\section{APPENDIX A: ADDITIONAL EXPERIMENTAL RESULTS OF WAVE-FRONT MODULATION}

We also provide the acoustic pressure patterns at other frequencies from $3000 \mathrm{~Hz}$ to $7000 \mathrm{~Hz}$, as given in Fig. 7 . It is seen that the metasurface is capable of working at any frequencies in this range.
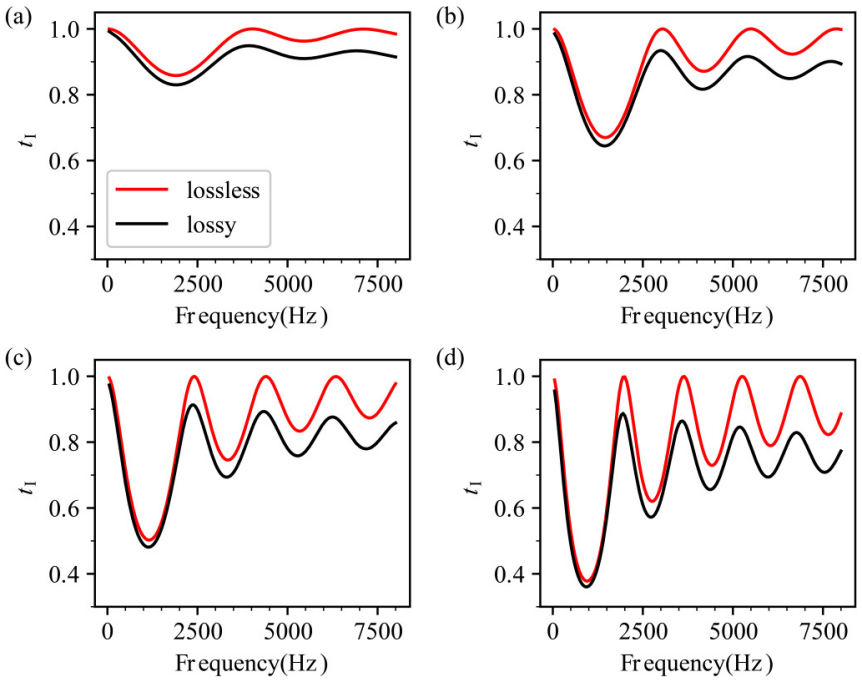

FIG. 9. Energy transmission coefficients of lossy gradient spiraling unit cells with different $l$. (a) $l=40 \mathrm{~mm}$, (b) $l=60 \mathrm{~mm}$, (c) $l=80 \mathrm{~mm}$, and (d) $l=100 \mathrm{~mm}$.

\section{APPENDIX B: THE EFFECT OF THERMAL AND VISCOUS LOSSES}

The complex refractive index of the uniform-helix-angle unit cells are retrieved based on a thermal-viscous acoustics
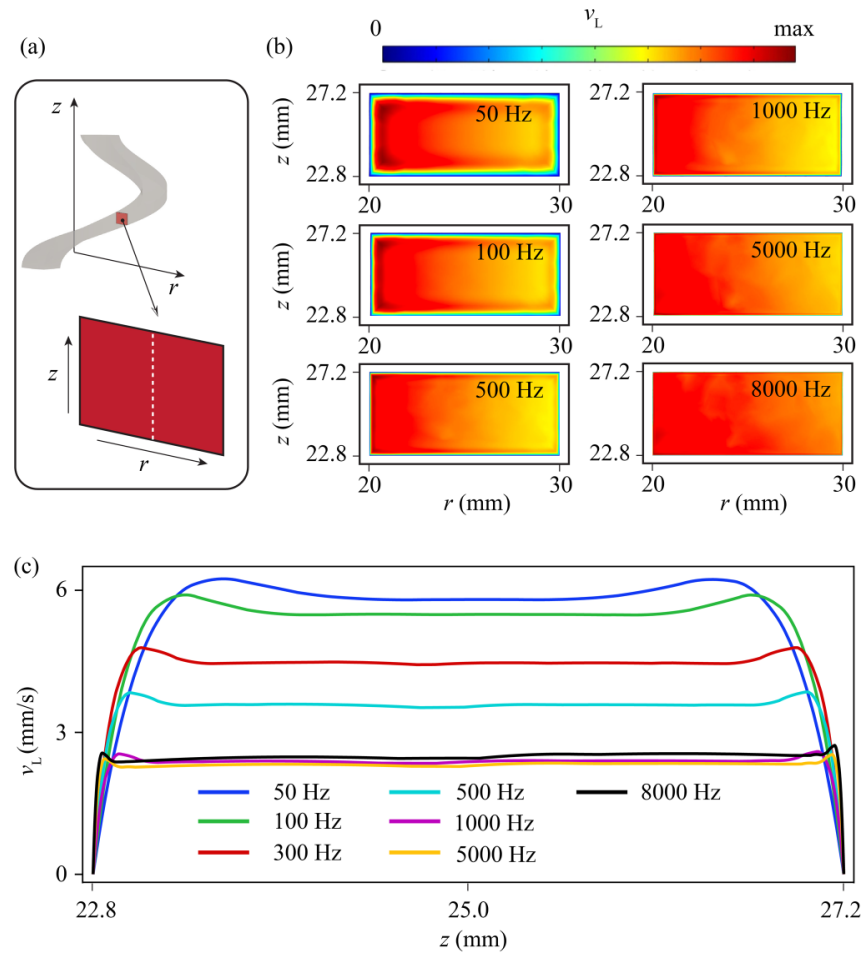

FIG. 10. The boundary layers of a gradient spiraling unit cell. (a) Schematic diagram of the cross-section area used in the simulations. The gradient tube is one channel of the impedance-matched unit cell shown in Fig. 3. The red rectangle is the intersection between $r-z$ plane and the gradient tube on its middle position. The dotted straight line is positioned at the center of the rectangle. (b) Local velocity maps of the cross-sectional rectangle in (a) at distinct frequencies. The results are normalized separately at each frequency. The horizontal and vertical coordinates are in the global coordinate system. (c) Distributions of local velocity along the white dotted line shown in (a). 
model, where the wavelength is longer than $7 h$. As shown in Fig. 8(a), the real-part refractive indices experience a relatively large dispersion at low frequencies. They coincide with the lossless results as the frequency goes higher. The coincidence shows the validity of the effective indices in the lossless case. The results of the imaginary part as shown in Fig. 8(b) indicate the decay of sound wave when propagating through the designed structures. For structures with larger $l$ values their imaginary effective indices are closer to 0 . On the one hand, more tortuous structure means smaller channels of wave propagation. The small area of cross section enhances the influence of thermal and viscous layers. On the other hand, large $l$ is related to high refractive index, which prolongs the attenuation distance of acoustic wave.

To evaluate the effect of the inherent thermal and viscous losses on the impedance-matched unit cells, a comparison of energy transmission between the lossless and lossy cases is shown in Fig. 9. The thermal and viscous losses can inevitably reduce the energy passing through the metamaterial. It is worth noting that the small shift of peaks and valleys between the lossless and lossy models indicates that the inherent losses only slightly affect the effective sound speed of the impedance matched spiraling metamaterials. The transmission coefficient of sound energy is more than 0.6 for all the unit cells with $l \leqslant 100 \mathrm{~mm}$.

The thickness of the viscous boundary layers inside the structures is also evaluated. As shown in Fig. 10, when the incident frequency is lower than $500 \mathrm{~Hz}$, a gradient distribution of the local velocity can be observed near the boundary of a spiraling channel. When the incident frequency is higher than $1000 \mathrm{~Hz}$, both the local velocity patterns and the solid lines illustrate much thinner boundary layers that would lead to rather weak influence on the wave propagation.
[1] S. A. Cummer, J. Christensen, and A. Alù, Controlling sound with acoustic metamaterials, Nat. Rev. Mater. 1, 16001 (2016).

[2] G. Ma and P. Sheng, Acoustic metamaterials: From local resonances to broad horizons, Sci. Adv. 2, e1501595 (2016).

[3] B. Assouar, B. Liang, Y. Wu, Y. Li, J. Cheng, and Y. Jing, Acoustic metasurfaces, Nat. Rev. Mater. 3, 460 (2018).

[4] A. Climente, D. Torrent, and J. Sánchez-dehesa, Sound focusing by gradient index sonic lenses, Appl. Phys. Lett. 97, 104103 (2010).

[5] T. P. Martin, M. Nicholas, G. J. Orris, L. W. Cai, D. Torrent, and J. Sánchez-Dehesa, Sonic gradient index lens for aqueous applications, Appl. Phys. Lett. 97, 113503 (2010).

[6] S. C. S. Lin, T. J. Huang, J. H. Sun, and T. T. Wu, Gradientindex phononic crystals, Phys. Rev. B 79, 094302 (2009).

[7] L. Zigoneanu, B. I. Popa, and S. A. Cummer, Design and measurements of a broadband 2D acoustic lens, Phys. Rev. B 84, 024305 (2011).

[8] T. M. Chang, G. Dupont, S. Enoch, and S. Guenneau, Enhanced control of light and sound trajectories with 3D gradient index lenses, New J. Phys. 14, 035011 (2012).

[9] N. Yu, G. Patrice, A. C. Mikhail, F. Aieta, T. Jean-Philippe, F. Capasso, and Z. Gaburro, Light propagation with phase discontinuities: generalized laws of reflection and refraction, Science 334, 333 (2011).

[10] H. Chen, Anomalous reflection of acoustic waves in air with metasurfaces at low frequency, Adv. Condens. Matter Phys. 2018, 5452071 (2018).

[11] Y. Li, X. Jiang, B. Liang, J. C. Cheng, and L. Zhang, Metascreen-Based Acoustic Passive Phased Array, Phys. Rev. Appl. 4, 024003 (2015).

[12] X. Jiang, Y. Li, B. Liang, J. C. Cheng, and L. Zhang, Convert Acoustic Resonances to Orbital Angular Momentum, Phys. Rev. Lett. 117, 034301 (2016).

[13] X. Yang, J. Yin, G. Yu, L. Peng, and N. Wang, Acoustic superlens using Helmholtz-resonator-based metamaterials, Appl. Phys. Lett. 107, 193505 (2015).

[14] K. Tang, C. Qiu, M. Ke, J. Lu, Y. Ye, and Z. Liu, Anomalous refraction of airborne sound through ultrathin metasurfaces, Sci. Rep. 4, srep06517 (2015).
[15] Y. Xie, W. Wang, H. Chen, A. Konneker, B. I. Popa, and S. A. Cummer, Wave-front modulation and subwavelength diffractive acoustics with an acoustic metasurface, Nat. Commun. 5, 5553 (2014).

[16] Y. Li, B. Liang, X. Tao, X. F. Zhu, X. Y. Zou, and J. C. Cheng, Acoustic focusing by coiling up space, Appl. Phys. Lett. 101, 233508 (2012).

[17] B. Yuan, Y. Cheng, and X. Liu, Conversion of sound radiation pattern via gradient acoustic metasurface with space-coiling structure, Appl. Phys. Express 8, 027301 (2015).

[18] Y. Li, X. Jiang, R. Q. Li, B. Liang, X. Y. Zou, L. L. Yin, and J. C. Cheng, Experimental Realization of Full Control of Reflected Waves with Subwavelength Acoustic Metasurfaces, Phys. Rev. Appl. 2, 064002 (2014).

[19] Z. Liang and J. Li, Extreme Acoustic Metamaterial by Coiling up Space, Phys. Rev. Lett. 108, 114301 (2012).

[20] X. Zhu, K. Li, P. Zhang, J. Zhang, C. Tian, J. Zhu, and S. Liu, Implementation of dispersion-free slow acoustic wave propagation and phase engineering with helical-structured metamaterials, Nat. Commun. 7, 11731 (2016).

[21] S. Zhao, A. Chen, Y. Wang, and C. Zhang, Continuously Tunable Acoustic Metasurface for Transmitted Wavefront Modulation, Phys. Rev. Appl. 10, 054066 (2018).

[22] S. Fan, S. Zhao, A. Chen, Y. Wang, B. Assouar, and Y. Wang, Tunable Broadband Reflective Acoustic Metasurface, Phys. Rev. Appl. 11, 044038 (2019).

[23] Y. Ding, E. C. Statharas, K. Yao, and M. Hong, A broadband acoustic metamaterial with impedance matching layer of gradient index, Appl. Phys. Lett. 110, 241903 (2017).

[24] K. Li, B. Liang, J. Yang, J. Yang, and J. chun Cheng, Acoustic broadband metacouplers, Appl. Phys. Lett. 110, 203504 (2017).

[25] K. Li, B. Liang, J. Yang, J. Yang, and J. chun Cheng, Broadband transmission-type coding metamaterial for wavefront manipulation for airborne sound, Appl. Phys. Express 11, 077301 (2018).

[26] S. Liang, T. Liu, F. Chen, and J. Zhu, Theoretical and experimental study of gradient-helicoid metamaterial, J. Sound Vib. 442, 482 (2019).

[27] Y. Xie, A. Konneker, B. Popa, S. A. Cummer, Y. Xie, A. Konneker, B. Popa, and S. A. Cummer, Tapered labyrinthine 
acoustic metamaterials for broadband impedance matching, Appl. Phys. Lett. 103, 201906 (2017).

[28] R. Ghaffarivardavagh, J. Nikolajczyk, R. Glynn Holt, S. Anderson, and X. Zhang, Horn-like space-coiling metamaterials toward simultaneous phase and amplitude modulation, Nat. Commun. 9, 1349 (2018).

[29] S. Tong, C. Ren, and W. Tang, Broadband extraordinary acoustic transmission via hornlike metamaterials, Appl. Phys. Express 11, 107302 (2018).

[30] G. Memoli, M. Caleap, M. Asakawa, D. R. Sahoo, B. W. Drinkwater, and S. Subramanian, Metamaterial bricks and quantization of meta-surfaces, Nat. Commun. 8, 14608 (2017).

[31] M. Molerón, M. Serra-Garcia, and C. Daraio, Acoustic Fresnel lenses with extraordinary transmission, Appl. Phys. Lett. 105, 114109 (2014).

[32] M. A. Norasikin, D. M. Plasencia, S. Polychronopoulos, G. Memoli, Y. Tokuda, and S. Subramanian, Soundbender: Dynamic acoustic control behind obstacles, in UIST 2018 Proceedings of the 31st Annual ACM Symposium on User Interface Software and Technology (ACM, New York, 2018), p. 247.
[33] K. Song, M. Anzan-Uz-Zaman, J.-H. Kwak, J.-Y. Jung, J. Kim, and S. Hur, Concentric artificial impedance surface for directional sound beamforming, AIP Adv. 7, 035315 (2017).

[34] Y. Li, G. Yu, B. Liang, X. Zou, G. Li, S. Cheng, and J. Cheng, Three-dimensional ultrathin planar lenses by acoustic metamaterials, Sci. Rep. 4, srep0 (2014).

[35] V. Romero-García, A. Cebrecos, R. Picó, V. J. SánchezMorcillo, L. M. Garcia-Raffi, and J. V. Sánchez-Pérez, Wave focusing using symmetry matching in axisymmetric acoustic gradient index lenses, Appl. Phys. Lett. 103, 264106 (2013).

[36] B. H. Song and J. S. Bolton, A transfer-matrix approach for estimating the characteristic impedance and wave numbers of limp and rigid porous materials, J. Acoust. Soc. Am. 107, 1131 (2000).

[37] V. Fokin, M. Ambati, C. Sun, and X. Zhang, Method for retrieving effective properties of locally resonant acoustic metamaterials, Phys. Rev. B 76, 144302 (2007).

[38] E. Kreyszig, Advanced Engineering Mathematics, $5^{\text {th }}$ ed. (Wiley, New York, 1983). 